\title{
Reações adversas ao benzonidazol no tratamento da Doença de Chagas: revisão sistemática de ensaios clínicos randomizados e controlados
}

\author{
Adverse reactions to benznidazole in the treatment of \\ Chagas' Disease: a systematic review of randomized and \\ controlled trials
}

\author{
Ariela Mota Ferreira' (1D, Renata Fiúza Damasceno ${ }^{1}$ (1), \\ Renato Sobral Monteiro-Junior ${ }^{1}$ (1), Igor Antônio Costa de Oliveira ${ }^{2}$ (1), \\ Thalita Emily Cezário Prates ${ }^{2}$ (1), Maria Carmo Pereira Nunes ${ }^{3}$ (1), \\ Desirée Sant'Ana Haikal ${ }^{1}$
}

\begin{abstract}
Resumo
Introdução: No Brasil, a única droga disponível para o tratamento específico do Trypanosoma cruzi, causador da Doença de Chagas (DC) é o Benzonidazol (BZN), cujas reações adversas podem propiciar a interrupção do tratamento. Objetivo: O objetivo desse trabalho é quantificar a proporção de ocorrência de efeitos adversos, descrevê-los e identificar os fatores de risco associados à essa ocorrência. Método: Revisão sistemática conduzida de acordo com os padrões estabelecidos pelo Preferred Reporting Items is Systematic Reviews and Metanalyses (PRISMA). Incluíram-se somente ensaios clínicos randomizados controlados. Resultados: Dos 134 estudos identificados, cinco foram incluídos. O tamanho da amostra variou de 77 a 2854 pessoas, e a faixa etária dos participantes, variou de zero a 75 anos. Todos os estudos incluídos apontaram reações adversas ao uso do BZN. A frequência de reação adversa chegou a $38 \%$ e a mais comum foi o rash cutâneo. $O$ abandono do tratamento devido à reação foi citado em três estudos, variando de 6,4\% a 13,4\%. Conclusão: As reações adversas ao uso do BZN variaram de acordo com a idade do paciente e esquema terapêutico de dose e tempo adotado, sendo o conhecimento médico acerca dessas reações imprescindível para seu uso seguro e adesão ao tratamento.

Palavras-chave: doença de Chagas; tripanossomicidas; efeitos colaterais e reações adversas relacionadas aos medicamentos; revisão sistemática; ensaio clínico.

\footnotetext{
Abstract

Background: In Brazil, the only drug available for the treatment of Trypanosoma cruzi, which causes Chagas' Disease (CD), is the Benznidazole (BZN) whose the adverse reactions might lead to treatment dropout. Objective: The objective of this study is to quantify the proportion of occurrence of adverse effects, to describe them and to identify the risk factors associated with this

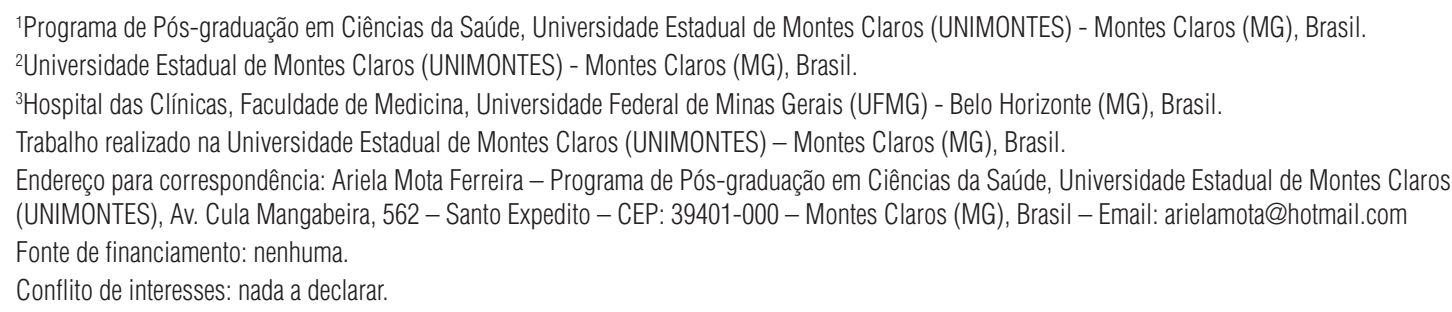


occurrence. Method: This is a systematic review conducted through the standards established by the Preferred Reporting Items in Systematic Reviews and Metanalyses (PRISMA). Only randomized controlled trials were included. Results: Five of 134 studies were included. The sample size ranged from 77 to 2854 people, and the age range of the participants ranged from zero to 75 years old. All included studies reported adverse reactions to BZN use. The frequency of adverse reaction was $38 \%$ and the most common reaction was the skin rash. The treatment dropout caused by the adverse reactions was cited in three studies and was ranging from $6.4 \%$ to $13.4 \%$. Conclusion: The adverse reactions caused by the use of BZN varied with the age of patients and the therapeutic scheme of dose and time adopted. Therefore, medical knowledge is imperative for the safe use of BZN and adherence to treatment. Keywords: Chagas disease; trypanosomicides; side effects and adverse drug reactions; systematic review; clinical trial.

\section{INTRODUÇÃO}

A Doença de Chagas (DC) é reconhecida pela Organização Mundial da Saúde (OMS) como uma das 17 doenças tropicais mais negligenciadas do mundo ${ }^{1}$. Causada pelo parasita Trypanosoma cruzi $^{2}$, a DC constitui um importante problema de saúde pública, sobretudo na América Latina, onde se estima que existam seis milhões de indivíduos infectados e 28 milhões sob o risco de contrair a infecção, com mais de 10 mil óbitos anuais ${ }^{1}$. No Brasil, há cerca de 2.500.000 indivíduos infectados pelo T. $c r u z i^{1-3}$. Apesar disso, as opções terapêuticas continuam limitadas e atualmente no mundo inteiro, dispõe-se apenas de dois fármacos para combater especificamente o parasita da DC: Benzonidazol (BZN) e Nifurtimox ${ }^{2,4}$.

No Brasil, a única droga disponível no momento para o tratamento específico da DC é o BZN, que teve os direitos e a tecnologia de fabricação cedida ao Brasil pela Roche ${ }^{5}$, sendo atualmente produzido e distribuído pelo Laboratório Farmacêutico de Pernambuco (LAFEPE). O Nifurtimox teve a sua comercialização interrompida na década de 1980, sendo disponibilizado pelo Ministério da Saúde (MS) em casos específicos de resistência ou graves efeitos colaterais associados ao $\mathrm{BZN}^{6,7}$. A dose recomendada do uso do $\mathrm{BZN}$ para adultos é de $5 \mathrm{mg} / \mathrm{kg} / \mathrm{dia}$, e para crianças $5-10 \mathrm{mg} / \mathrm{kg} / \mathrm{dia}$, ambas por via oral, durante 60 dias. $\mathrm{O}$ medicamento pode ser obtido pelo Sistema Único de Saúde (SUS), e é contraindicado para gestantes ou mulheres em idade fértil e que não estejam em uso de contraceptivos ${ }^{8}$.

Não há dúvidas sobre a indicação desse medicamento na fase aguda da doença, que é definida pela evidência do T. cruzi no exame direto do sangue periférico. O tratamento, nesse caso, deve ser realizado logo após a confirmação diagnóstica, independentemente da via de transmissão? .

Quanto ao uso do medicamento na fase crônica, recomenda-se que as crianças, com idade igual ou inferior a 12 anos, devam ser todas tratadas. No entanto, para os adultos, o tratamento antiparasitário deve ser considerado de forma individualizada, mas não deve ser realizado em indivíduos na fase crônica da DC com a forma cardíaca grave, uma vez que não há evidências de benefícios clínicos na evolução desses pacientes ${ }^{8}$. Alguns estudos observacionais têm demonstrado que o tratamento antiparasitário é capaz de diminuir a progressão da doença em proporção expressiva dos $\operatorname{casos}^{10,11}$. Em um estudo prospectivo, multicêntrico e randomizado, duplo-cego e controlado com placebo, não foi evidenciada diferença significativa entre os dois grupos, que apresentaram cardiopatia chagásica crônica; mas detectou redução da carga parasitária por meio da reação em cadeia da polimerase no sangue do grupo tratado com $\mathrm{BZN}^{12}$.

Embora o uso clínico do BZN seja considerado seguro ${ }^{13}$, efeitos adversos relativamente frequentes com o seu uso têm sido descritos na literatura ${ }^{8,12}$. Dentre os efeitos adversos mais graves apontados estão a dermatopatia alérgica, depressão da medula óssea e a polineuropatia periférica, que em alguns casos, pode acarretar a interrupção do tratamento ${ }^{14}$. Dessa forma, é de extrema importância que o médico esteja ciente das potenciais reações adversas à droga, bem como sobre sua frequência e tempo de ocorrência ${ }^{13}$.

Assim, o objetivo desse estudo foi realizar uma revisão sistemática de ensaios clínicos randomizados e controlados (ECRC), a fim de responder à pergunta de pesquisa de acordo com a estratégia PICOS ${ }^{15}$ : qual a proporção de ocorrência, a que estão relacionados e quais são os efeitos adversos manifestados por usuários do BZN durante o tratamento da DC?

\section{MÉTODO}

Foi conduzida uma revisão sistemática de ensaios clínicos randomizados controlados, de acordo com os padrões estabelecidos pelo Preferred Reporting Items for Systematic Reviews and Metanalyses (PRISMA) ${ }^{16}$, acerca das reações adversas ao BZN no tratamento da DC.

\section{Critérios de elegibilidade dos estudos}

Foram considerados elegíveis para o presente estudo somente ensaios clínicos randomizados e controlados realizados em humanos sobre o uso isolado do BZN (sem estar associado a outra medicação) na quimioterapia da DC, publicados nos últimos 25 anos. Essa escolha foi devida à disponibilidade de artigos e ao maior número de estudos publicados sobre o tema nesse período, e com publicações nos idiomas inglês, português e espanhol. Foram excluídos os estudos com ausência de grupo controle, outros que não informaram sobre os efeitos adversos e sobre a dose do medicamento usada, e aqueles que não foram localizados por inconsistência na sua referência, tornando o artigo indisponível nas bases de dados conhecidas. 


\section{Recursos utilizados e estratégias de busca}

Os artigos foram pesquisados, de março de 2017 a janeiro de 2018, nas bases de dados SCIELO, LILACS, Portal de Periódicos da CAPES, MEDLINE, PubMed e Cochrane Library por meio de descritores listados em Descritores de Ciências em Saúde (DeCS) e no Medical Subject Headings (MeSH), buscando-os pelo título e/ou resumo dos artigos. A seleção inicial foi realizada pela leitura do título e resumo, e quando os artigos foram considerados insuficientes para tal definição, os mesmos foram lidos na íntegra. As etapas de busca e seleção foram realizadas de forma independente por dois pesquisadores, e as discordâncias entre eles foram resolvidas por um terceiro pesquisador mediante discussão, até obtenção de consenso.

$\mathrm{Na}$ busca dos artigos, utilizaram-se de forma combinada os termos: "doença de chagas", "tripanossomicidas", "ensaio clínico", "chagas disease", "trypanosomicides" e "clinical trial", sendo que as chaves de busca foram elaboradas de acordo com cada base de dados. Para a busca dos artigos na base de dados PubMed, o descritor "chagas disease" indexado pelo $\mathrm{MeSH}$ foi associado ao termo "benznidazole", classificado como Supplementary Concept; filtrando a busca para ensaios clínicos. Dessa forma, a estratégia de busca final utilizada foi: "Benznidazole"[Supplementary Concept] AND "Chagas Disease"[Mesh] AND “Clinical Trial” [ptyp] OR “Controlled clinical trial" [ptyp] OR "Randomized Controlled trial" [ptyp]. Nas demais bases de dados, a utilização dos recursos boleanos AND, OR, title/abstract bem como a estratégia foi adaptada de acordo com a disponibilidade de busca a partir dos termos presentes no MeSH ou em seus correspondentes no DeCS ("Doença de Chagas" para "Chagas Disease" e "benzonidazol" para "Benznidazole"), de forma a garantir a obtenção do maior número de ensaios clínicos possíveis.

\section{Seleção dos estudos}

A seleção dos estudos respeitou duas fases: 1) leitura dos títulos e resumos; e 2) leitura do texto na íntegra. Na primeira fase, foram selecionados os artigos com pelo menos dois dos termos principais da busca explícitos no título ou seus sinônimos, identificados no MeSH (para os estudos em inglês) ou em DeCS (para os estudos em português e espanhol), quando possível. Avaliou-se a duplicidade de artigos, sendo considerada só uma versão. Resumos foram avaliados na sequência a fim de confirmarem adequação temática. Esta etapa inicial foi realizada por dois revisores independentes, que classificaram os estudos como incluído, excluído ou duvidoso. Aqueles classificados como duvidosos foram revistos e discutidos por um terceiro revisor, para obtenção de um consenso. Assim, os artigos que atenderam aos critérios de elegibilidade seguiram para a fase seguinte. Na segunda fase, relacionada àleitura do texto na íntegra, foi realizada a confirmação da adequação temática e o respeito aos critérios de elegibilidade. Os estudos que atenderam a tais critérios (informações sobre efeitos adversos, sobre a dose do BZN utilizada e presença de grupo controle) foram incluídos. Os estudos incluídos foram utilizados para a extração de informações/dados e seguiram para uma última fase, em que foi realizada uma análise qualitativa dos estudos.

Além das bases descritas, foram pesquisadas referências considerando a literatura cinzenta, relatórios de pesquisa, documentos governamentais, e também artigos identificados nas listas de referências de outras revisões ou de estudos originais relacionados ao tema.

\section{Principais dados extraídos}

Dos estudos incluídos foram extraídos como dados: autores do estudo; ano de publicação; número de participantes (n); faixa etária; fase da doença; tempo de uso do medicamento; dose do medicamento empregada; número e percentual de abandono permanente da droga devido à reação adversa; número e percentual de reações adversas e as principais conclusões. A sumarização das informações coletadas foi organizada em tabelas, e os dados das mesmas foram todos apresentados conforme tinham sido disponibilizados nas publicações originais (Tabelas 1 e 2). As conclusões apresentadas referiram-se à transcrição literal de trechos que faziam menção às reações adversas.

\section{Avaliação da qualidade dos estudos}

Para avaliação da qualidade metodológica dos ensaios clínicos randomizados controlados foi utilizada a Escala de Jadad ${ }^{17}$. Essa escala consta de tópicos centrados na validade interna da pesquisa, considerando randomização, mascaramento duplo-cego, perda e exclusão de participantes, que estão diretamente relacionadas com a redução de vieses. Para cada critério atendido (resposta "sim") foi atribuído um ponto na classificação, e um ponto adicional se os métodos de randomização e mascaramento fossem descritos de forma adequada. Assim, a pontuação máxima passível de ser obtida pela escala é de cinco (05) pontos, sendo considerado de boa qualidade aqueles que recebem três (03) ou mais pontos. Essa etapa não foi considerada como critério de inclusão. Esse processo de avaliação qualitativa foi realizado por dois revisores independentes. Somente para os casos em que não houve consenso, um terceiro revisor foi incluído e esse revisor foi responsável pela decisão final.

\section{RESULTADOS}

Na Figura 1 é apresentado o fluxo de seleção dos artigos. A busca bibliográfica resultou em 134 artigos. Desses artigos, 78 foram excluídos por duplicidade. Dos 56 artigos restantes, 18 foram excluídos por não serem ensaios clínicos, sete por não abordarem o BZN como monoterapia, 11 por não abordarem o BZN, nove por terem sido publicados há mais de 25 anos, quatro por não informarem sobre os efeitos adversos, um por 

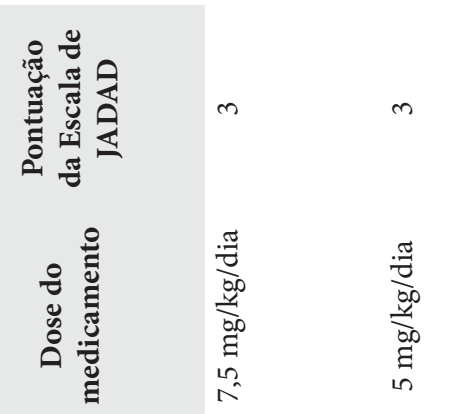

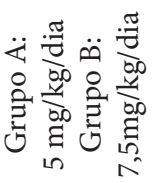

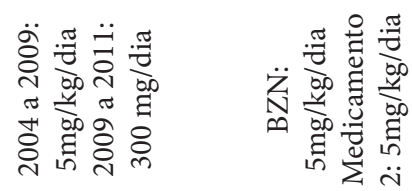

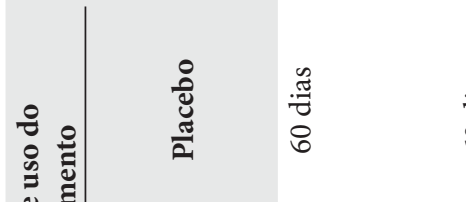

:

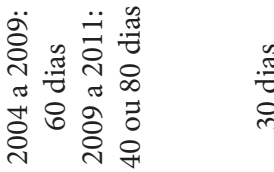

窟

Z

蒁

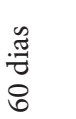

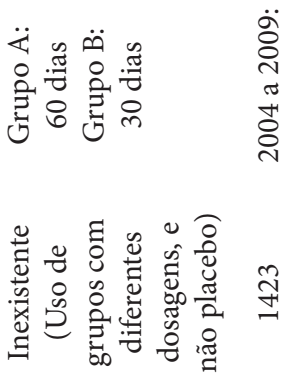

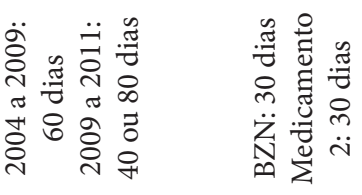

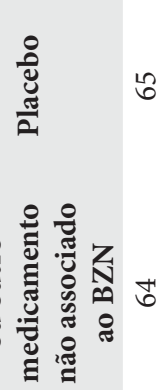

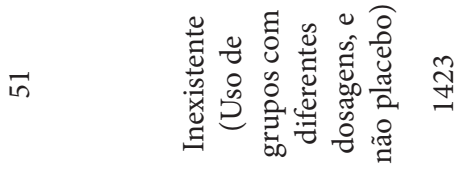

$\stackrel{8}{3}$

ב

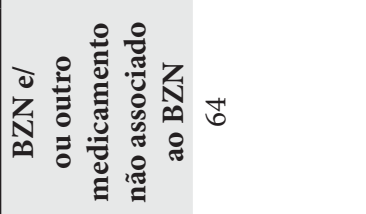

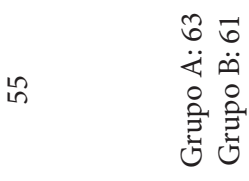

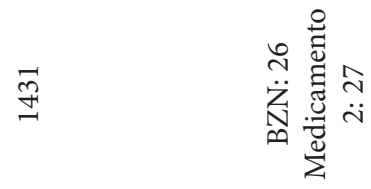

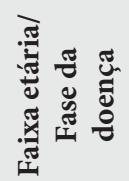

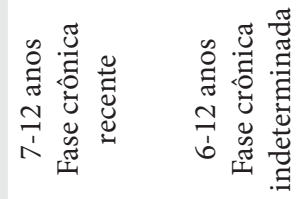

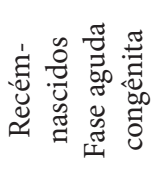

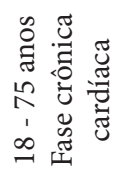

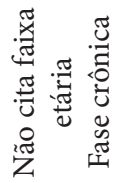

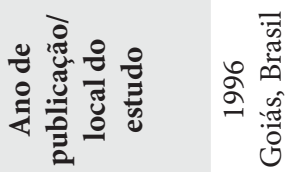

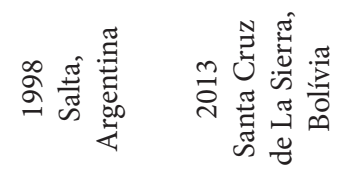

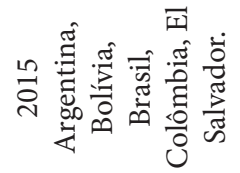

ล人苛
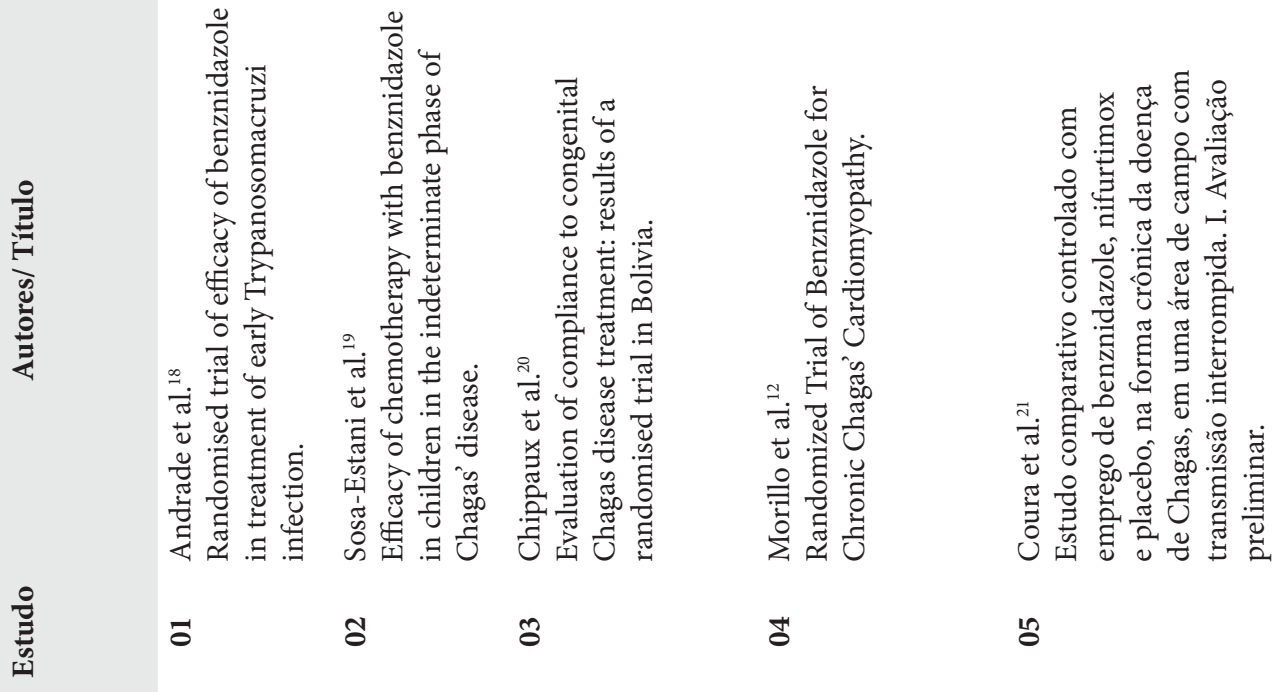

ช

\%

莎

$\ddot{8}$

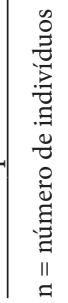




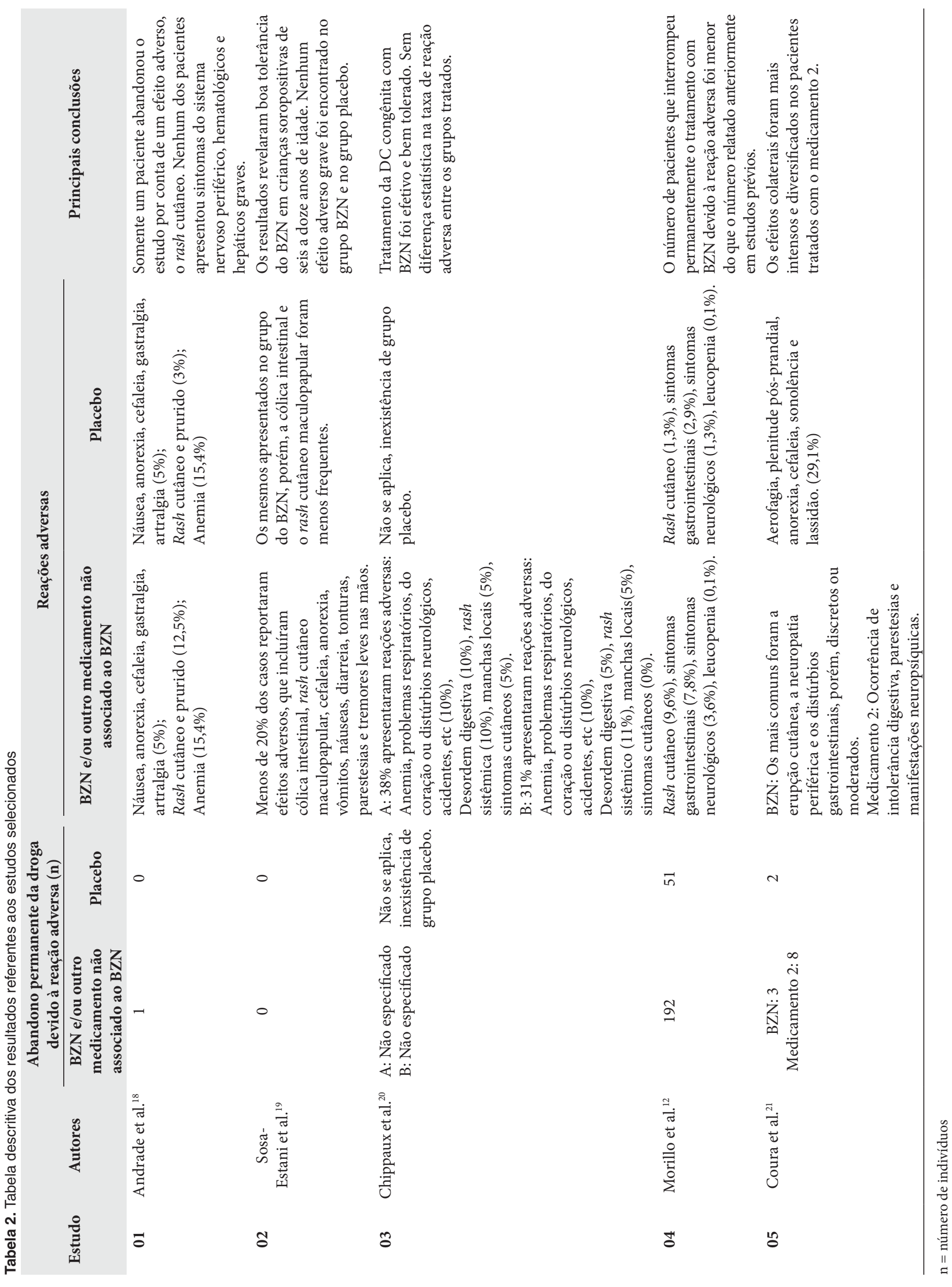


não informar a dose do medicamento adotada, e por fim mais um artigo que não foi localizado por inconsistência na sua referência. Assim, 56 artigos foram triados, mas 51 foram excluídos por não atenderem aos critérios de elegibilidade (Figura 1).

Conforme a Tabela 1, dos cinco estudos que atenderam aos critérios de inclusão desta revisão, dois foram publicados nos últimos cinco anos. O tamanho da amostra variou de 77 a 2854 pessoas. O score de qualidade dos estudos, segundo a Escala de Jadad, variou de três a quatro pontos, sendo que: um estudo não citou o termo "randomizado" mesmo apresentando a descrição desse processo; dois artigos não esclareceram sobre o método de randomização; um outro artigo não relatou se o estudo foi duplo-cego; e quatro não citaram o método para mascaramento, ou o método citado foi considerado inadequado.

A faixa etária dos participantes, dentre os estudos que a citaram, variou de zero a 75 anos, sendo que a maioria dos estudos envolveram crianças e pessoas na fase crônica da DC. O tempo de uso do medicamento variou de 30 a 80 dias e a dosagem de 5,0 mg/kg/dia a 7,5 mg/ $\mathrm{kg} / \mathrm{dia}$, ou, quando em dose diária, $300 \mathrm{mg} / \mathrm{dia}$.

A Tabela 2 apresenta os principais resultados e conclusões referentes às reações adversas dos estudos selecionados. Em todos os estudos houve registro de reações adversas ao BZN. A taxa de reações adversas chegou a $38 \%$ e o desfecho mais comum foi o rash cutâneo. $\mathrm{O}$ abandono do tratamento devido a essas
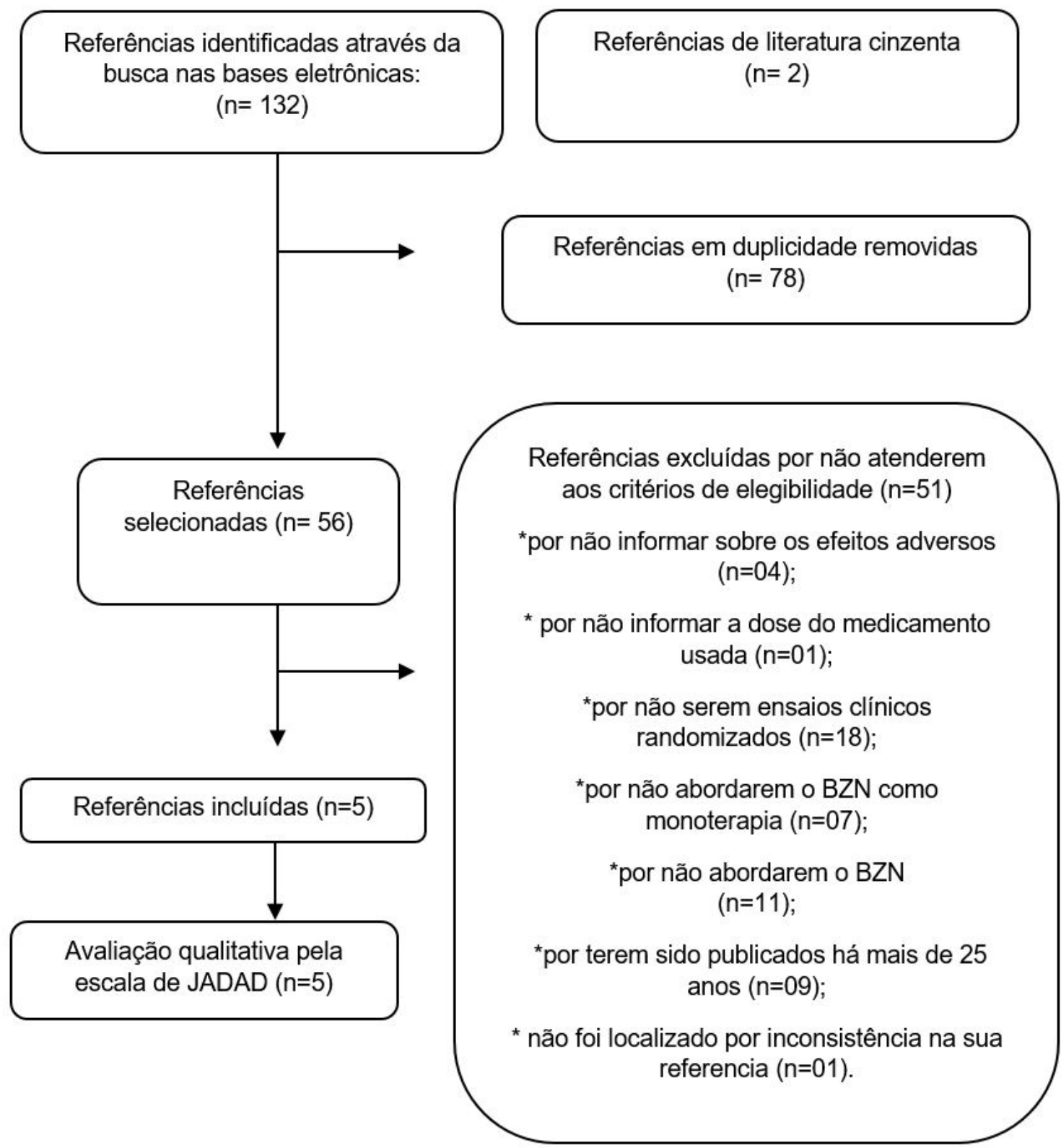

Figura 1. Fluxo de seleção dos artigos da Revisão Sistemática. 
reações também foi citado em todos os cinco estudos, variando de $6,4 \%$ a $13,4 \%$ (Tabela 2 ).

\section{DISCUSSÃO}

A maioria dos estudos incluídos nessa revisão sistemática foi publicada em revistas internacionais, sendo quatro em língua inglesa e um em português, e os estudos foram realizados na América Latina entre os anos de 1996 e 2015; reafirmando assim a atualidade do tema. A taxa geral de reação adversa foi citada por dois estudos, variando de 19\% (taxa citada em "menos de 20\%" de forma textual) a $38 \%$. A prevalência de cada reação adversa, ou grupo de reação adversa, foi citada em três estudos, sendo a mais prevalente o rash cutâneo, alcançando $12,5 \%$ dos usuários do BZN. Quando avaliados pela Escala de Jadad, com valores de zero a cinco (0-5), os estudos obtiveram nota entre três e quatro.

Estudos anteriores avaliaram a eficácia da terapia, comparando-se com placebo, outro medicamento ou grupos de diferentes dosagens de BZN; porém, nenhum estudo foi conduzido com o propósito de avaliar a reação adversa ao uso do BZN. Assim, as reações adversas foram mencionadas de forma secundária com metodologias de apresentação muitas vezes pouco detalhadas e não padronizadas. $\mathrm{O}$ número de participantes, a dosagem adotada e o tempo de uso de BZN no grupo experimental, bem como a composição dos grupos de comparação variaram amplamente nos estudos, e nesse caso, dificultou as comparações entre seus resultados e impediu a condução de metanálises. Essa variabilidade implicou na impossibilidade de esclarecimento de qual método de administração do BZN diminuiria suas reações adversas, considerando a dose, tempo de uso, ou a idade do paciente, desse modo dificultando a interpretação do perfil de segurança do BZN.

Nesta revisão, ou seja, nos cinco estudos incluídos, houve o registro de reações adversas ao uso do BZN. A aplicação clínica do BZN exige atenção às suas várias reações adversas, sendo um procedimento seguro se o médico estiver ciente das mesmas ${ }^{22}$. O Consenso Brasileiro em Doença de Chagas de 2005 relatou a ocorrência de reações adversas em cerca de 30\% dos usuários de BZN, considerando todas as faixas etárias e fases da doença9

Em crianças, o esquema terapêutico recomendado é de 5 a $10 \mathrm{mg} / \mathrm{kg} / \mathrm{dia}$, durante 60 dias consecutivos, fracionando-se a dose diária em duas ou três tomadas antes da alimentação ${ }^{23}$. Essa recomendação foi seguida pelos três estudos presentes nessa revisão que relataram o tratamento em crianças e recém-nascidos. Somente um estudo incluído relatou a avalição das reações adversas em adultos e/ou idosos ${ }^{12}$, pois outro estudo que avaliou a fase crônica não indicou a idade dos participantes ${ }^{21}$. Considerando os estudos que citaram o abandono devido à reação adversa e à faixa etária dos participantes ${ }^{12,18,19}$, observou-se que a taxa foi menor entre os estudos realizados com crianças. Esse resultado corrobora com estudos prévios que afirmaram que a ocorrência de reação adversa é proporcional à idade, sendo mais bem tolerado em indivíduos mais novos ${ }^{8,24}$.

Em relação à fase da doença, os estudos incluídos trataram das fases aguda congênita e crônica. Um artigo incluído avaliou o uso do BZN na fase aguda congênita e nesse artigo, foram abordadas duas posologias diferentes quanto à dose e ao tempo de tratamento. Embora os autores tivessem verificado taxas menores de reações adversas no esquema de menor tempo de tratamento com maior dosagem diária, do que no esquema de maior tempo de tratamento com dosagens diárias menores, não houve diferença estatística na taxa de reação adversa entre os grupos tratados ${ }^{20}$. Estudos prévios conceituais têm afirmado que o uso do BZN durante a fase aguda da DC apresentaria significativa redução da ocorrência de reações adversas quando comparados ao uso na fase crônica da $\mathrm{DC}^{25,26}$. Entretanto, ao se comparar as taxas de ocorrência de reações adversas ao BZN, considerando dois ECRC incluídos nessa revisão, verificou-se menor taxa de reações adversas entre crianças na fase crônica da $\mathrm{DC}^{19}$, quando comparada com a taxa verificada para recém-nascidos na fase aguda ${ }^{20}$. Esse dado controverso merece melhor elucidação, uma vez que as dosagens e tempo de uso foram similares em ambos os estudos.

Foram incluídos quatro estudos que abordaram a fase crônica, sendo um estudo da fase crônica recente ${ }^{18}$, um outro estudo da fase crônica indeterminada ${ }^{19}$, e dois estudos da fase crônica $\operatorname{tardia}^{12,21}$. O estudo de Andrade et al..$^{18}$ foi conduzido com crianças até 12 anos, na fase crônica recente da DC, independentemente se as mesmas apresentaram ou não repercussões clínicas da DC, tais como: cardiopatia chagásica crônica; alterações no eletrocardiograma; megaesôfago; entre outras. Esse estudo verificou maior taxa de abandono do tratamento com BZN devido às reações adversas quando comparado com as taxas verificadas no estudo de Sosa-Estani et al. ${ }^{19}$, realizado com crianças de faixa etária similar; porém na fase crônica indeterminada, ou seja, sem qualquer repercussão clínica presente. Embora não seja possível inferir se essa diferença nas taxas de abandono seria ou não significativa, há de considerar a possibilidade de tal diferença estar relacionada à fase e progressão da doença, ou mesmo à maior dose utilizada no estudo por Andrade et al. ${ }^{18}$.

A fase crônica indeterminada tem particular relevância por ser a forma clínica de maior prevalência na DC. O tratamento antiparasitário específico, isto é, com o BZN, pode ser indicado na maioria dos casos dessa fase ${ }^{1}$. O ECRC conduzido entre crianças nessa fase apresentou taxa de abandono do tratamento devido às reações adversas igual a zero ${ }^{19}$, mas cabe ressaltar que a dose utilizada foi a menor proposta para o tratamento9.

A prescrição do BZN durante a fase crônica tardia da DC é controversa na literatura cientifica ${ }^{12,27,28}$. Dois ECRC foram conduzidos nessa fase ${ }^{12,21}$. 
Para tentar diminuir o dilema, foi realizada uma investigação multicêntrica internacional, randomizada, duplo-mascarada e controlada por placebo, avaliando-se a evolução clínica por seis anos de pacientes na fase crônica tratados com $\mathrm{BZN}^{12}$. Esse ECRC conduzido com 2854 pacientes com cardiopatia chagásica crônica, sendo a maioria de nacionalidade brasileira, evidenciou maior taxa de conversão negativa da expressão PCR no grupo tratado com o BZN quando comparado com o grupo tratado com placebo. Cerca de 13,4\% dos pacientes descontinuaram permanentemente o tratamento com o BZN devido aos efeitos adversos. Segundo os autores, a taxa identificada foi menor do que a taxa relatada anteriormente em outros estudos pequenos e observacionais ${ }^{12}$. O outro ECRC conduzido na fase crônica da $\mathrm{DC}^{21}$ também apresentou conclusão favorável ao BZN quanto às reações adversas. Os autores concluíram que, ao se comparar com o outro medicamento disponível para o tratamento antiparasitário da DC, a ocorrência de reação adversa no grupo BZN foi menor ${ }^{21}$.

Cabe ressaltar que não foi identificado nenhum estudo analisando o tratamento com BZN em mulheres grávidas. $\mathrm{O}$ uso de BZN na gravidez não é recomendado devido à falta de evidência de segurança completa para efeitos potencialmente teratogênicos ${ }^{29,30}$. Apesar disso, há relatos de situações especiais em que o tratamento com BZN teve que ser prescrito no último trimestre da gravidez devido ao risco de vida que a mãe apresentava durante fase aguda da infecção por T. cruzi $i^{29,30}$.

As reações adversas podem ser minimizadas, atenuadas ou melhor controladas quando se adota o esquema terapêutico que apresenta menos problemas de hipersensibilidade, no caso dos adultos $5 \mathrm{mg} / \mathrm{kg} / \mathrm{dia}$, por via oral, em duas ou três tomadas diárias, durante 60 dias $^{8,28}$. Doses maiores aumentam a frequência e a intensidade de reações adversas e podem impossibilitar o tratamento, por isso a dose máxima recomendada, em todos os casos, é de $300 \mathrm{mg} / \mathrm{dia}^{31}$. De acordo com o II Consenso Brasileiro em Doença de Chagas, quando a dose diária ultrapassar os $300 \mathrm{mg}$, recomenda-se estender o tempo de tratamento até alcançar a dose total calculada para 60 dias $^{8}$. O ECRC que avaliou o tratamento em adultos ${ }^{12}$ seguiu tais recomendações, variando o tempo de uso do medicamento. Inicialmente adotou a dosagem de $5 \mathrm{mg} / \mathrm{kg} /$ dia por 60 dias. Nos três últimos anos do estudo, a dose foi alterada para $300 \mathrm{mg} /$ dia por 40 ou 80 dias, de acordo com o peso do paciente ${ }^{12}$.
Essa revisão apresentou alguns pontos fortes que merecem destaque: pesquisa bibliográfica abrangente e sistemática; utilização de critérios de elegibilidade explícitos e reprodutíveis; e apresentação do padrão de qualidade alcançado pelos estudos incluídos. Por outro lado, a existência de poucos ECRC identificados, o fato dessa temática abordada não ser o foco principal de tais estudos e a falta de padronização na apresentação dos desfechos investigados, além de terem impossibilitado a condução de metanálises, representaram pontos de fragilidade dessa revisão. Além disso, o viés de publicação sempre representa uma ameaça metodológica à validade dos resultados considerando essa metodologia de pesquisa. Apesar de tais limitações, acredita-se que os resultados possibilitaram identificar o atual e real conhecimento com base em evidências disponíveis acerca das reações adversas ao uso do BZN, tema que tem sido, de certa forma, negligenciado ou pouco investigado na literatura científica.

A partir dos resultados dessa revisão, constatou-se a ocorrência de reações adversas ao uso do BZN, com variações de acordo com o esquema terapêutico e a idade do paciente. Foi possível observar menor taxa de abandono do tratamento com o BZN devido às reações adversas nos casos crônicos de crianças até 12 anos utilizando menor dose do medicamento, e/ou nos casos onde o tratamento foi iniciado com menor idade. O rash cutâneo foi identificado como a reação adversa ao uso do BZN mais frequente.

Nenhum estudo incluído foi conduzido especificamente com o propósito de avaliar tais reações, visto que essas reações foram apresentadas de forma secundária e pouco detalhadas. Assim, não foi possível identificar o esquema terapêutico que minimizasse a ocorrência de reações adversas ao uso BZN devido à falta de padronização na apresentação dos desfechos investigados e às inconsistências nas apresentações dos dados dos ECRC incluídos. Desse modo, considerando que o conhecimento médico acerca dessas reações é imprescindível para seu uso seguro e redução de seu abandono, enfatiza-se a necessidade de condução de mais ECRC voltados especificamente ao estudo de tais reações; a fim de gerar maior evidência científica quanto à sua prevalência, aos esquemas de administração do BZN e às fases da doença que poderiam minimizar sua ocorrência.

\section{REFERÊNCIAS}

1. World Health Organization. Chagas disease in Latin America: an epidemiological update based on 2010 estimates. Wkly Epidemiol Rec. 2015;90(6):33-43. PMid:25671846.

2. Amato V No, Pasternak J. Centenário da doença de Chagas. Rev Saude Publica. 2009;43(2):381-2. http://dx.doi.org/10.1590/S0034-89102009000200022. PMid:19287879.

3. Santos JP, Lima-Costa MF, Peixoto SV. Aspectos nutricionais associados à infecção crônica pelo Trypanosoma cruzi (Chagas 1909) entre idosos:

Projeto Bambuí. Cad Saude Publica. 2013;29(6):1141-8. http://dx.doi. org/10.1590/S0102-311X2013000600011. PMid:23778546.

4. Tarleton RL, Reithinger R, Urbina JA, Kitron U, Gürtler RE. The challenges of Chagas disease: grim outlook or glimmer of hope? PLoS Med. 2007;4(12):e332. http://dx.doi.org/10.1371/journal.pmed.0040332. PMid:18162039.

5. Cançado JR. Tratamento etiológico da doença de Chagas pelo benznidazole. In: Brener Z, Andadre ZA, Barral-Netto M, editores. Trypanosoma cruzi 
e doença de Chagas. 2. ed. Rio de Janeiro: Guanabara Koogan; 2000. p. 389-405.

6. Pontes VM, Souza AS Jr, Cruz FM, Coelho HL, Dias AT, Coêlho IC, et al. Reações adversas em pacientes com doença de Chagas tratados com benzonidazol, no Estado do Ceará. Rev Soc Bras Med Trop. 2010;43(2):1827. http://dx.doi.org/10.1590/S0037-86822010000200015. PMid:20464150.

7. Oliveira MF, Nagao-Dias AT, Pontes VMO, Souza AS Jr, Coelho HLL, Coêlho ICB. Tratamento etiológico da doença de Chagas no Brasil. Rev Patol Trop. 2008;37(3):209-28. http://dx.doi.org/10.5216/rpt.v37i3.5063.

8. Dias JCP, Ramos JRN Jr, Gontijo ED, Luquetti A, Shikanai-Yasuda MA, Coura JR, et al. II Consenso Brasileiro em Doença de Chagas, 2015. Epidemiol Serv Saude. 2016;25(N. esp.):7-86. PMid:27869914.

9. Brasil. Ministério da Saúde. Secretaria de Vigilância em Saúde. Consenso Brasileiro de Doença de Chagas. Rev Soc Bras Med Trop. 2005;38(Supl 3):7-29.

10. Rassi A Jr, Rassi A, Marin-Neto JA. Chagas disease. Lancet. 2010;375(9723):1388402. http://dx.doi.org/10.1016/S0140-6736(10)60061-X. PMid:20399979.

11. Fabbro DL, Streiger ML, Arias ED, Bizai ML, Del Barco M, Amicone NA. Trypanocide treatment among adults with chronic Chagas disease living in Santa Fe City (Argentina), over a mean follow-up of 21 years: parasitological, serological and clinical evolution. Rev Soc Bras Med Trop. 2007;40(1):1-10. http://dx.doi.org/10.1590/S0037-86822007000100001. PMid:17486245.

12. Morillo CA, Marin-Neto JA, Avezum A, Sosa-Estani S, Rassi A Jr, Rosas F, et al. Randomized trial of benznidazole for chronic Chagas cardiomyopathy. N Engl J Med. 2015;373(14):1295-306. http://dx.doi.org/10.1056/NEJMoa1507574. PMid:26323937.

13. Cançado JR. Long term evaluation of etiological treatment of Chagas disease with benznidazole. Rev Inst Med Trop São Paulo. 2002;44(1):29-37. http:// dx.doi.org/10.1590/S0036-46652002000100006. PMid:11896410.

14. Marin-Neto JA, Rassi A Jr, Avezum A Jr, Mattos AC, Rassi A, Morillo $\mathrm{CA}$, et al. The BENEFIT trial: testing the hypothesis that trypanocidal therapyis beneficial for patients with chronic Chagas heart disease. Mem Inst Oswaldo Cruz. 2009;104(Supl 1):319-24. http://dx.doi.org/10.1590/ S0074-02762009000900042. PMid:19753491.

15. Santos CMC, Pimenta CAM, Nobre MRC. A estratégia PICO para a construção da pergunta de pesquisa e busca de evidências. Rev. LatinoAm. Enfermagem. 2007;15(3):508-11. http://dx.doi.org/10.1590/S010411692007000300023.

16. Moher D, Shamseer L, Clarke M, Ghersi D, Liberati A, Petticrew M, et al. Preferred reporting items for systematic review and meta-analysis protocols (PRISMA-P) 2015 statement. Syst Rev. 2015;1(4):1. http://dx.doi. org/10.1186/2046-4053-4-1. PMid:22587946.

17. Jadad AR, Moore RA, Carroll D, Jenkinson C, Reynolds DJ, Gavaghan DJ, et al. Assessing the quality of reports of randomized clinical trials: is blinding necessary. Control Clin Trials. 1996;17(1):1-12. http://dx.doi. org/10.1016/0197-2456(95)00134-4. PMid:8721797.

18. Andrade AL, Zicker F, Oliveira RM, Almeida Silva S, Luquetti A, Travassos LR, et al. Randomised trial of efficacy of benznidazole in treatment of early Trypanosomacruzi infection. Lancet. 1996;348(13):1407-13. http://dx.doi. org/10.1016/S0140-6736(96)04128-1. PMid:8937280.
19. Sosa-Estani S, Porcel BM, Segura EL, Yampotis C, Ruiz AM, Velazquez E. Efficacy of chemotherapy with benznidazole in children in the indeterminate phase of Chagas' disease. Am J Trop Med Hyg. 1998;59(4):526-9. http:// dx.doi.org/10.4269/ajtmh.1998.59.526. PMid:9790423.

20. Chippaux JP, Salas-Clavijo AN, Postigo JR, Schneider D, Santalla JA, Brutus L. Evaluation of compliance to congenital Chagas disease treatment: results of a randomised trial in Bolivia. Trans R Soc Trop Med Hyg. 2013;107(1):17. http://dx.doi.org/10.1093/trstmh/trs004. PMid:23296694.

21. Coura JR, Abreu LL, Willcox HP, Petana W. Estudo comparativo controlado com emprego de benznidazole, nifurtimox e placebo, na forma crônica da doença de Chagas, em uma área de campo com transmissão interrompida. I. Avaliação preliminar. Rev Soc Bras Med Trop. 1997;30(2):139-44. http:// dx.doi.org/10.1590/S0037-86821997000200009. PMid:9148337.

22. Fragata AA, Silva MAD, Boainain E. Tratamento etiológico da doença de Chagas na fase aguda e crônica. Rev. Soc. Cardiol. 1994;4(7):192-7.

23. Dias JCP, Coura JR. Clínica e terapêutica da doença de Chagas: uma abordagem prática para o clínico geral. Rio de Janeiro: FIOCRUZ; 1997. http://dx.doi.org/10.7476/9788575412435.

24. Pérez-Molina JA, Pérez-Ayala A, Moreno S, Fernández-González MC, Zamora J, López-Velez R. Use of benznidazole to treat chronic Chagas' disease: a systematic review with a meta-analysis. J Antimicrob Chemother. 2009;64(6):1139-47. http://dx.doi.org/10.1093/jac/dkp357. PMid:19819909.

25. Menezes C, Costa CG, Gollob KJ, Dutra WO. Clinical aspects of Chagas disease and implications for novel therapies. Drug Dev Res. 2011;72(6):4719. http://dx.doi.org/10.1002/ddr.20454. PMid:22267887.

26. Pinazo MJ, Muñoz J, Posada E, Lopez-Chejade P, Gállego H, Ayala $\mathrm{E}$, et al. Tolerance of benznidazole in treatment of Chagas' disease in adults. Antimicrob Agents Chemother. 2010;54(11):4896-9. http://dx.doi. org/10.1128/AAC.00537-10. PMid:20823286.

27. Andrade JP, Marin-Neto JA, Paola AA, Vilas-Boas F, Oliveira GM, Bacal F. I Latin American guidelines for the diagnosis and treatmentof Chagas' heart disease. Arq Bras Cardiol. 2011;96(6):434-42. http://dx.doi.org/10.1590/ S0066-782X2011000600002. PMid:21789345.

28. Viotti R, Vigliano C, Lococo B, Bertocchi G, Petti M, Alvarez MG, et al. Long-term cardiac outcomes of treating chronic chagas disease with BZN e versus no treatment. Ann Intern Med. 2006;144(34):724-34. http://dx.doi. org/10.7326/0003-4819-144-10-200605160-00006. PMid:16702588.

29. Soares JL So, Medeiros FPM, La Roca MF, Silva KER, Lima LNA, Rolim PJ No. Delineamento de alternativas terapêuticas para o tratamento da Doença de Chagas. Rev Patol Trop. 2007;36(2):103-18. http://dx.doi.org/10.5216/ rpt.v36i2.1783.

30. Sosa-Estani S. Transmissão congênita de Tripanosoma cruzi na Argentina. Rev Soc Bras Med Trop. 2005;38(2 Supl 2):29-32. PMid:16482810.

31. Brasil. Ministério da Saúde. Secretaria de Vigilância em Saúde. Departamento de Vigilância Epidemiológica. Guia de vigilância epidemiológica. 7. ed. Brasília: Ministério da Saúde; 2017. 